\title{
Einwirkung von Natrium in flüssigem Ammoniak auf die Buntesalz-A-Kette von Schweineinsulin
}

\author{
Y. SHIMONISHI * und H. ZaHN \\ Deutsches Wollforschungsinstitut an der Technischen Hochschule Aachen \\ W. Puls \\ Institut für Pharmakologie der Farbenfabriken Bayer AG, Wuppertal \\ (Z. Naturforschg. 24 b, 422-427 [1969] ; eingegangen am 11. September 1968)
}

\begin{abstract}
In connection with synthetic work on insulin a study was made of the behaviour of natural A-chain-Buntesalt isolated from pig insulin after treatment with sodium in liquid ammonia. The A-chain was partially degradated and compounds with different amino acid compositions were built. It was possible to isolate the remaining intact A-chain as tetra-S-sulfonate by chromatography on DEAE-Sephadex with a yield of 55 per cent. This fraction gave, after recombination with isolated B-chain, the same yield of insulin as did A-chain undamaged by sodium.
\end{abstract}

Bei allen bisher publizierten Synthesen der Insulin-A-Kette ${ }^{1-7}$ wurden die Cysteinreste als $S$-Benzylcystein eingebaut und die Ketten zur Abspaltung der Benzylschutzgruppe der Einwirkung von Natrium in flüssigem Ammoniak ${ }^{8}$ unterworfen. Bei dieser Reduktion erfolgt nach KATSOYANNIS ${ }^{9}$ eine teilweise Entschwefelung. Die anschließende oxydative Sulfitolyse ergibt ein Gemisch von Ketten mit unterschiedlichem Gehalt an Thiosulfatgruppen. Wir haben kürzlich ein Verfahren zur Gewinnung reiner Tetrathiosulfat-A-Kette durch Ionenaustausch-Chromatographie an DEAE-Sephadex beschrieben ${ }^{10}$. So konnte z. B. das aus homogener $S$-Benzyl-Kette, die nach der Methode von Merrifield synthetisiert worden war $^{5}$, nach Natriumreduktion und oxydativer Sulfitolyse erhaltene Produkt in acht verschiedene Fraktionen aufgetrennt werden, von denen nur eine

* Jetzt Institute for Protein Research, Osaka University, Osaka, Japan.

1 J. Meienhofer, E. Schnabel, H. Bremer, O. Brinkhoff, R. Zabel, W. SRoka, H. Klostermeyer, D. Brandenburg, T. Okuda u. H. Zahn, Z. Naturforschg. 18 b, 1120 [1963].

2 P. G. Katsoyannis, K. Funuda, A. Tometsko, K. Suzuki u. M. Tilak, J. Amer. chem. Soc. 86, 930 [1964].

3 Y.-T. Kung, Y.-c.Du, W.-T. Huang, C.-C. Chen, L.-T. Ke, S.-C. Hu, R.-Q. Jiang, S.-Q. Chu, C.-I. NiU, J.-Z. Hsu, W.-c. Chang, L.-L. Cheng, H.-s. Li, Y. Wang, T.-P. LoH, A.-H. Chi, C.-H. LI, P.-T. SHI, Y.-H. YieH, K.-L. TANG u. C.-Y. HsING, Scientia sinica 14, 1710 [1965].

4 A. Marglin u. R. B. Merrifield, J. Amer. chem. Soc. 88, 5051 [1966].

5 H. Zahn, T. Okuda u. Y. Shimonishi, Peptides, Proceedings of the Eighth European Peptide Symposium, Noordwijk, The Netherlands, September 1966, North-Holland Publishing Company, Amsterdam 1967, p. 108; Angew. Chem. 79, 424 [1967]. reinem $\mathrm{A}\left(\mathrm{SSO}_{3}{ }^{\ominus}\right)_{4} *$ entsprach, das mit $10-20 \%$ Ausbeute isoliert wurde. Dieses Verfahren bot uns jetzt die Möglichkeit, an der Buntesalz-A-Kette zu untersuchen, ob die Entschwefelung durch das Reduktionssystem Natrium/Ammoniak bedingt ist oder von der Art der zu spaltenden Schwefelbindung abhängt. Tsou et al. ${ }^{11}$ schlossen aus dem Ergebnis von Rekombinations-Versuchen, daß die Natriumbehandlung von Insulin-Thiosulfatketten nicht $\mathrm{zu}$ einer Schädigung der Ketten führt.

Aus Schweineinsulin durch oxydative Sulfitolyse dargestelltes $\mathrm{A}\left(\mathrm{SSO}_{3}{ }^{\ominus}\right)_{4}$ wurde als Ausgangsmaterial für die vorliegende Studie gewählt. Dieses Präparat wurde mit Natrium in flüssigem Ammoniak behandelt, das Reduktionsprodukt erneut einer oxydativen Sulfitolyse unterworfen und hierauf das rohe Sulfitolyseprodukt an DEAE-Sephadex frak-

${ }^{6}$ U. Weber, F. Schneider, P. Köhler u. G. Weitzel, Hoppe-Seyler's Z. physiol. Chem. 348, 947 [1967]; U. WEber, S. Hörnle, G. Grieser, K.-H. Herzog u. G. Weitzel, 348, 1715 [1967]; U. Weber, S. Hörnle, P. Köhler, G. Nagelschneider, K. Eisele u. G. Weitzel, 349, 512 [1968].

7 S. Hörnle, Hoppe-Seyler's Z. physiol. Chem. 348, 1355 [1967].

8 R. H. Siffert u. V. du Vigneaud, J. biol. Chemistry $\mathbf{1 0 8 ,}$ 753 [1935].

9 P. G. Katsoyannis, Diabetes 13, 339 [1964], Amer. J. Med. 40, 652 [1966].

10 Y. Shimonishi u. H. Zahn, Biochim. biophysica Acta [Amsterdam] 154, 598 [1968].

* A $\left(\mathrm{SSO}_{3}{ }^{\ominus}\right)_{4}$ bedeutet das S-Sulfonat-Derivat der A-Kette des Schweineinsulins, $\mathrm{B}\left(\mathrm{SSO}_{3}{ }^{\ominus}\right)_{2}$ das der B-Kette des Rinderinsulins.

11 C.-L. Tsou, Y.-c. Du u. G.-J. Xü, Scientia Sinica 10, 332 [1961]. 
tioniert. Von den einzelnen Fraktionen wurde das elektrophoretische Verhalten und die AminosäureZusammensetzung bestimmt; die insulinbildende Potenz wurde durch Kombination mit reiner InsulinB-Kette nach der Methode von Du et al. ${ }^{12}$ ermittelt.

\section{Material und Methoden}

Schweineinsulin wurde uns großzügigerweise vom Nordisk Insulinlaboratorium als Geschenk überlassen.

\section{DEAE-Sephadex A-25}

Das von der Pharmacia Uppsala bezogene Gel besaß eine Korngröße von $40-120 \mu$ und eine Säurebindung von 3,5 $\pm 0,5$ Milliäquivalenten/g.

Oxydative Sulfitolyse von Schweineinsulin

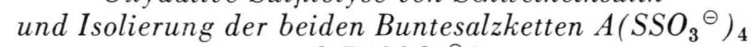
und $\mathrm{B}\left(\mathrm{SSO}_{3}{ }^{\ominus}\right)_{2}$

$1 \mathrm{~g}$ krist. Schweineinsulin wurde nach DRECHSEL ${ }^{13}$ in einem Gemisch von $35 \mathrm{ml} 0,05 \mathrm{M}$ Natriumacetat, $25 \mathrm{ml}$ Dioxan und $70 \mathrm{mg}$ Dinatriumäthylendiamintetraacetat $(\mathrm{pH} 7,5)$ mit 2,24 g Natriumsulfit und 4,8 g Natriumtetrathionat bei $37^{\circ}$ umgesetzt. Nach einer Reaktionszeit von 90 Min. wurde die Lösung 5 Stdn. lang gegen dest. Wasser dialysiert (Viskingschlauch 20/32). Der Inhalt des Dialyseschlauches wurde gefriergetrocknet und das gebildete Pulver in Phosphatpuffer $\left(0,1 \mathrm{M} \mathrm{NaH}{ }_{2} \mathrm{PO}_{4}, 0,1 \mathrm{M} \mathrm{Na}{ }_{2} \mathrm{PO}_{4}, 8 \mathrm{M}\right.$ Harnstoff $)$ gelöst und nach BAILEY und COLE ${ }^{14}$ durch Chromatographie an Dowex $50 \times 2$ in $\mathrm{A}\left(\mathrm{SSO}_{3}{ }^{\ominus}\right)_{4}$ und $\mathrm{B}\left(\mathrm{SSO}_{3}{ }^{\ominus}\right)_{2}$ getrennt.

Die Fraktionen, welche die Buntesalzketten enthielten, wurden getrennt jeweils 5 Stdn. gegen dest. Wasser dialysiert und der Inhalt des Dialyseschlauches gefriergetrocknet. Zur Abtrennung von Salzen diente die Gelfiltration von Lösungen der Rohpräparate in $0,1 \mathrm{M} \mathrm{Am}$ moniumhydrogencarbonat an Sephadex G-25 (20× $470 \mathrm{~mm})$. Ausbeute an $\mathrm{A}\left(\mathrm{SSO}_{3}{ }^{\ominus}\right)_{4} 280 \mathrm{mg}(60 \% \mathrm{~d}$. Th.) und an $\mathrm{B}\left(\mathrm{SSO}_{3}{ }^{\ominus}\right)_{2} 460 \mathrm{mg}(75 \% \mathrm{~d}$. Th.).

Die Proteinkonzentration der isolierten Präparate wurde durch Vergleich ihrer Absorptionsmaxima bei $275 \mathrm{~nm}$ im Beckman-Spektrophotometer DK-2 zu

$$
\begin{aligned}
& 100 \% \pm 5 \% \text { für } \mathrm{A}\left(\mathrm{SSO}^{\ominus}\right)_{4} \text {, und } \\
& 101 \% \pm 5 \% \text { für } \mathrm{B}\left(\mathrm{SSO}_{3}\right)_{2} \text { bestimmt. }
\end{aligned}
$$

\section{Enzymatische Hydrolyse}

$1 \mu \mathrm{Mol}$ Substrat wurde bei $37^{\circ} 24 \mathrm{Stdn}$. lang mit $400 \mu \mathrm{g}$ Aminopeptidase-M ${ }^{15}$ (Röhm und Haas GmbH., Darmstadt) in $0,01 \mathrm{~m}$ Trispufferlösung ( $\mathrm{pH} \mathrm{8,0)} \mathrm{inku-}$

12 Y.-C. Du, R.-Q. Jiang u. C.-L. Tsou, Scientia sinica 14, 229 [1965].

13 H. Zahn u. E. Drechsel, Hoppe-Seyler's Z. physiol. Chem. 349, 359 [1968].

14 J. L. Bailey, Biochem. J. 67, 21 P [1957]; J. L. Bailey u. R. D. Cole, J. biol. Chemistry 234, 1733 [1959]. biert. Das Enzym besaß eine Aktivität von 10000 Einheiten. Nach Denaturieren des Enzyms mit 50-proz. Trichloressigsäure wurde das Hydrolysat nach SPACKMAN, MOORE und STEIN ${ }^{16}$ analysiert.

\section{Resynthese von Insulin aus Buntesalzketten}

Die Rekombination der Ketten wurde unter Anlehnung an die Vorschrift von Du et al. ${ }^{12}$ ausgeführt. Ein Gemisch von $8,1 \mathrm{mg} \mathrm{A}\left(\mathrm{SSO}_{3}{ }^{\ominus}\right)_{4}$ und $7,1 \mathrm{mg} \mathrm{B}\left(\mathrm{SSO}_{3}{ }^{\ominus}\right)_{2}$, zusammen 15,2 mg Protein, wurde mit $1 \mathrm{M}$ Thioglykolsäure-Lösung vom $\mathrm{pH} 5$ bei Zimmertemperatur $18 \mathrm{Stdn}$. lang behandelt. Das Gemisch der reduzierten Ketten wurde mit Essigsäure auf $\mathrm{pH} 3,8$ eingestellt und die Fällung in $2,5 \mathrm{ml}$ Wasser suspendiert und mit $1 \mathrm{~N}-\mathrm{Na}$ tronlauge alkalisch ( $\mathrm{pH}$ 10,6) gelöst. Anschließend wurde mit $0,1 \mathrm{~m}$ Glycinpuffer $(\mathrm{pH} \quad 10,6)$ auf $3,0 \mathrm{ml}$ aufgefüllt. Die alkalische Lösung wurde bei $0-3{ }^{\circ} \mathrm{C}$ 24. Stdn. aufbewahrt. Nach der Autoxydation der SHGruppen der Insulinketten wurde das Oxydationsprodukt durch Gelfiltration über Sephadex G-25 $(20 \times 470$ $\mathrm{mm}$ ) entsalzt. Nach Gefriertrocknung der Lösung in $0,1 \mathrm{~m}$ Ammoniumhydrogencarbonat resultierten 11,3 $\mathrm{mg}$ festes Resyntheseprodukt.

\section{Bestimmung des Insulingehaltes in den Oxydations- produkten}

Die Oxydationsprodukte wurden im Vergleich zu kristallinem Insulin auf ihre Blutzucker-senkende Wirkung an nüchternen Ratten untersucht ${ }^{17}$. Ihr Insulingehalt wurde im 4-Punkte-Test ermittelt.

Der wahre Insulingehalt kann zwischen dem halben und dem doppelten Wert liegen. Reines $\mathrm{A}\left(\mathrm{SSO}_{3}{ }^{\ominus}\right)_{4}$ und $\mathrm{B}\left(\mathrm{SSO}_{3}{ }^{\ominus}\right)_{2}$ wurden in 15 verschiedenen Ansätzen miteinander kombiniert und folgende Werte erhalten: Tab. 1.

\section{Behandlung von $\mathrm{A}\left(\mathrm{SSO}_{3}{ }^{\ominus}\right)_{4}$ mit Natrium in flüssigem Ammoniak und Fraktionerung des Sulfitolyseproduktes}

$80,8 \mathrm{mg} \mathrm{A}\left(\mathrm{SSO}_{3}{ }^{\ominus}\right)_{4}$ aus Schweineinsulin, zuvor über $\mathrm{P}_{2} \mathrm{O}_{5}$ im Vakuum bei $60^{\circ}$ über Nacht getrocknet, wurden in $80 \mathrm{ml}$ frisch über Natrium dest. flüssigem Ammoniak gelöst und bei $-70^{\circ}$ mit $20-25$ mg Natriumstückchen 3,5 Min. lang behandelt, bis eine leicht blaue Farbe einige Sek. lang bestehen blieb. Die Lösung wurde durch Zugabe von Ammoniumchlorid entfärbt und sofort lyophilisiert. Das dabei gewonnene feste reduzierte A-Kettenpräparat

15 G. Pfleiderer, P. G. Celliers, N. Stanulović, E. D. Wachsmuth, H. Determann u. G. Braunitzer, Biochem. Z. 340, 552 [1964].

${ }^{16}$ D. H. Spackman, W. H. Stein u. S. Moore, Analyt. Chem. 30, 1190 [1958].

17 H. Zahn, E. Drechsel u. W. Puls, Hoppe-Seyler's Z. physiol. Chem. 349, 385 [1968]. 


\begin{tabular}{|c|c|c|c|c|c|c|c|}
\hline $\begin{array}{l}\text { Prä- } \\
\text { parat } \\
\text { Nr. }\end{array}$ & $\begin{array}{c}\mathrm{A}\left(\mathrm{SSO}_{3}{ }^{\ominus}\right)_{4} \\
{[\mathrm{mg}]}\end{array}$ & $\begin{array}{c}\mathrm{B}\left(\mathrm{SSO}_{3}{ }^{\ominus}\right)_{2} \\
{[\mathrm{mg}]}\end{array}$ & $\begin{array}{l}\text { Oxyd } \\
\text { [mg] }\end{array}$ & $\begin{array}{l}\text { produkt } \\
\text { Ausbeute } \\
\text { in \% von } \\
(\mathrm{A}+\mathrm{B}) \\
(x)\end{array}$ & $\begin{array}{l}\text { Gefundener } \\
\text { in } \mathrm{E} / \mathrm{mg} \\
(\mathrm{Y})\end{array}$ & $\begin{array}{l}\text { Insulingehalt } \\
\text { in } \% \\
\begin{array}{l}(=\mathrm{Y} / 25 \cdot 100)(\mathrm{W} \\
\\
+\end{array}\end{array}$ & $\begin{array}{c}\text { Insulin- } \\
\text { ausbeute* } \\
\mathrm{W}=\mathrm{Z} \cdot \mathrm{X} / 100) \\
\text { bezogen auf } \\
\mathrm{A}\left(\mathrm{SSO}_{3}{ }^{\ominus}\right)_{4} \\
+\mathrm{B}\left(\mathrm{SSO}_{3}{ }^{\ominus}\right)_{2}\end{array}$ \\
\hline $\mathrm{S} 2$ & 5,28 & 4,63 & 9,1 & $(100)$ & 1,92 & $(7,68)$ & 7,68 \\
\hline S 3 & 8,21 & 7,15 & 11,2 & $(79,4)$ & 0,31 & $(1,24)$ & 1,02 \\
\hline S 5 & 8,18 & 7,15 & 11,3 & $(80,8)$ & 0,66 & $(2,64)$ & 2,13 \\
\hline S 8 & 8,07 & 7,16 & 12,2 & $(87,8)$ & 0,65 & $(2,60)$ & 2,28 \\
\hline S 10 & 8,12 & 7,18 & 11,0 & $(78,6)$ & 1,95 & $(7,80)$ & 6,13 \\
\hline S 13 & 8,11 & 7,12 & 10,9 & $(78,4)$ & 1,65 & $(6,60)$ & 5,17 \\
\hline S 15 & 8,09 & 7,11 & 10,6 & $(76,3)$ & 1,45 & $(5,80)$ & 4,43 \\
\hline S 17 & 8,14 & 7,11 & 11,9 & $(85,0)$ & 0,96 & $(3,84)$ & 3,26 \\
\hline S 19 & 8,17 & 7,12 & 11,8 & $(84,3)$ & 0,76 & $(3,04)$ & 2,56 \\
\hline S 21 & 8,14 & 7,13 & 11,7 & $(83,6)$ & 1,11 & $(4,44)$ & 3,71 \\
\hline S 23 & 8,13 & 7,15 & 11,2 & $(80,8)$ & 2,13 & $(8,52)$ & 6,82 \\
\hline S 25 & 8,11 & 7,16 & 11,7 & $(83,6)$ & 2,90 & $(11,6)$ & 9,50 \\
\hline S 27 & 8,12 & 7,14 & 11,3 & $(80,7)$ & 3,66 & $(14,6)$ & 11,7 \\
\hline S 39 & 8,11 & 7,12 & 12,3 & $(88,5)$ & 5,55 & $(22,2)$ & 19,6 \\
\hline $\mathrm{S} 41$ & 8,20 & 7,13 & 12,2 & $(87,2)$ & 3,38 & $(13,5)$ & 11,8 \\
\hline
\end{tabular}

Tab. 1. Insulinausbeuten bei der Kombination von $\mathrm{A}\left(\mathrm{SSO}_{3}{ }^{\ominus}\right)$, mit $\mathrm{B}\left(\mathrm{SSO}_{3} \ominus\right)$, nach der Methode von Du et al. ${ }^{12}$. ${ }^{*} \mathrm{KATSOY}$ ANNIS u. Tometsкo ${ }^{18}$ beziehen die Insulinausbeuten auf die Menge an $\mathrm{B}\left(\mathrm{SSO}_{3}{ }^{\ominus}\right)_{2}$ allein. Man hätte auch hier alle Ausbeuten auf die B-Kette umrechnen können. Bei der von Katsoyannis und Tometsko modifizierten A-Kettenüberschuß-Methode von Du et al. werden 5 Äquivalente A-Kette auf 1 Äquivalent B-Kette verwendet, bei der chinesischen Originalmethode ${ }^{12}$, die in der vorliegenden Arbeit angewendet wurde, jedoch nur 1,5 Äquivalente A-Kette. Die Berechnung auf B-Kette erhöht die rechnerischen Insulin-Ausbeuten im Falle der $\mathrm{K}$ a $\mathrm{t}$ s o y a $\mathrm{n}$ n i s - Methode auf etwa den dreifachen Wert des Insulingehaltes der Präparate, bei einem Molverhältnis von 1,5 zu 1 nur auf das 1,2-fache. Im Hinblick auf das präparative Ziel unserer Arbeiten geben wir die Insulinausbeuten so an, daß ersichtlich ist, wieviel Gew.-\% aus dem Gemisch der Buntesalzketten in Insulin umgewandelt wurde.

wurde einer oxydativen Sulfitolyse unterworfen, wobei man dies mit $0,25 \mathrm{~g}$ Natriumsulfit und $0,25 \mathrm{~g}$ Natriumtetrathionat in $2,39 \mathrm{~g}$ Guanidin- $\mathrm{HCl}$ enthaltendem Wasser (Totalvolumen $5 \mathrm{ml}$ ) bei $\mathrm{pH} 6$ bei Zimmertemperatur 20 Stdn. reagieren ließ. Der Sulfitolyseansatz wurde durch Gelfiltration über Sephadex G-25 $(2 \times 47 \mathrm{~cm})$ von Salzen befreit, und die Proteinlösung gefriergetrocknet. Man erhielt $77,8 \mathrm{mg}$ Sulfitolyseprodukt. Dieses Präparat wurde durch Chromatographie an DEAE-Sephadex nach der früher beschriebenen Methode ${ }^{10}$ fraktioniert.

Wie aus Abb. 1 hervorgeht, ließ sich das Sulfitolyseprodukt der mit Natrium reduzierten A-Kette in 6 Fraktionen auftrennen. Und zwar wurden aus 41,9 mg Rohprodukt 2,3 mg Fraktion I, 1,4 mg II, $2,5 \mathrm{mg}$ III, $3,9 \mathrm{mg} \mathrm{IV}, 23,3 \mathrm{mg}$ V ( $=55,6 \%$ Ausbeute) und 4,6 mg Fraktion VI, zusammen $38 \mathrm{mg}$ oder $90 \%$ des Sulfitolyseproduktes, isoliert.

Die Hauptfraktion V wurde von der DEAESephadexsäule mit dem 0,2 N Salzsäure - 8 m Harnstoffpuffer eluiert, verhielt sich also chromatographisch genau wie authentisches $\mathrm{A}\left(\mathrm{SSO}_{3}{ }^{\ominus}\right)_{4}$. Dies würde bedeuten, daß $\mathrm{A}\left(\mathrm{SSO}_{3}{ }^{\ominus}\right)_{4}$ nach Einwirkung

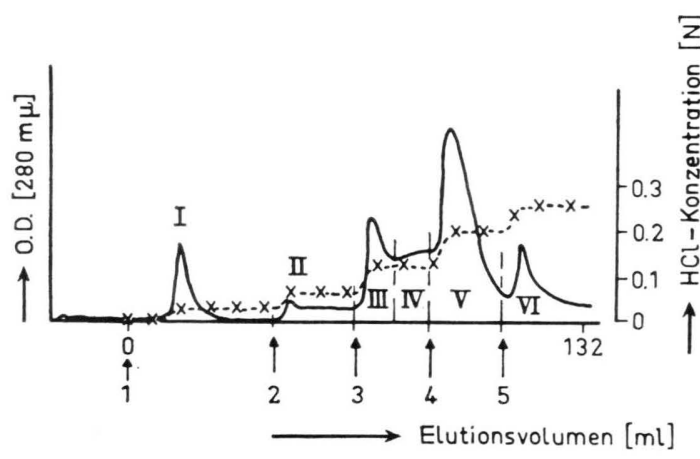

Abb. 1. DEAE-Sephadex-Chromatogramm des Sulfitolyseproduktes von mit Natrium in flüssigem Ammoniak behandeltem $\mathrm{A}\left(\mathrm{SSO}_{3}{ }^{\ominus}\right)_{4}$. Stufenweise Elution. - : Proteinabsorption bei $280 \mathrm{~nm} . \cdots \times \cdots \times$ : Konzentration der Salzsäure im Eluat. $\uparrow$ : Pufferwechsel. 1. $3,06 \cdot 10^{-2} \mathrm{~N} \mathrm{HCl}-8 \mathrm{M}$ Harnstoff, 2. $6,50 \cdot 10^{-2} \mathrm{~N} \mathrm{HCl}-8 \mathrm{M}$ Harnstoff, 3. $1,35 \cdot 10^{-1} \mathrm{~N} \mathrm{HCl}-$ $8 \mathrm{M}$ Harnstoff, 4. $1,99 \cdot 10^{-1} \mathrm{~N} \mathrm{HCl}-8 \mathrm{M}$ Harnstoff, 5 . $2,65 \cdot 10^{-1} \mathrm{~N} \mathrm{HCl}-8 \mathrm{M}$ Harnstoff.

von Natrium in flüssigem Ammoniak und anschließender oxydativer Sulfitolyse wieder zu 56\% regeneriert werden kann und daß die fünf an DEAE-Sephadex abgetrennten Fraktionen nur Nebenprodukte sind. 
Um festzustellen, welchen Einfluß die Behandlung mit Ammoniak allein auf die regenerierbare Ausbeute an $\mathrm{A}\left(\mathrm{SSO}_{3}{ }^{\ominus}\right)_{4}$ ausübt, wurden $72,3 \mathrm{mg}$ getrocknetes $\mathrm{A}\left(\mathrm{SSO}_{3}{ }^{\ominus}\right)_{4}$ nur mit flüssigem Ammoniak ohne Zugabe von Natrium behandelt. 71,8 mg Sulfitolyseprodukt wurden wiedergewonnen.

Auch dieses Präparat wurde durch DEAE-Sephadex-Chromatographie fraktioniert. Wie Abb. 2 zeigt, überwiegt hier die mit $0,2 \mathrm{~N}$ Salzsäure eluierbare Fraktion V noch stärker als in Abb. 1. 23,9 mg dieser Fraktion konnten ausgehend von $35,4 \mathrm{mg}$ Sulfitolyseprodukt isoliert werden. Dies sind 67,5 Prozent. Die übrigen Fraktionen wurden in so geringen Mengen erhalten, daß sie nicht näher analysiert werden konnten.

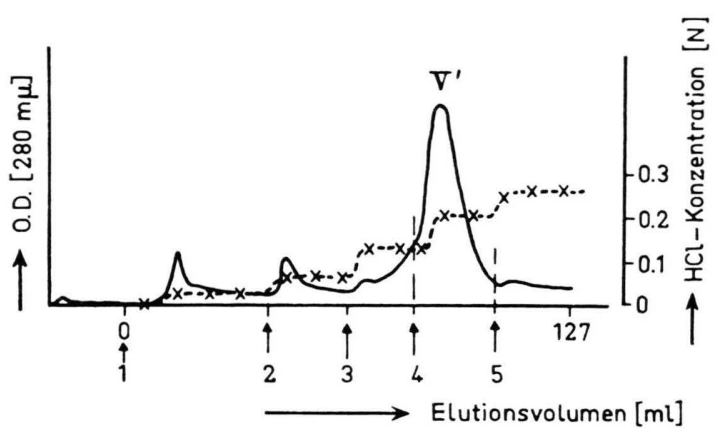

Abb. 2. DEAE-Sephadex-Chromatogramm des Sulfitolyseproduktes von nur mit flüssigem Ammoniak behandeltem $\mathrm{A}\left(\mathrm{SSO}_{3}{ }^{\ominus}\right)_{4}$. Stufenweise Elution mit Salzsäure in $8 \mathrm{M}$ Harnstoff. 1 : UV-Absorption bei $280 \mathrm{~nm} . \cdots \times \cdots \times \cdots \times$ : Konzentration der Salzsäure im Eluat. $\uparrow$ : Pufferwechsel. 1. $3,06 \cdot 10^{-2} \mathrm{~N} \mathrm{HCl}-8 \mathrm{M}$ Harnstoff, $2.6,50 \cdot 10^{-2} \mathrm{~N} \mathrm{HCl}-$ $8 \mathrm{~m}$ Harnstoff, 3. $1,35 \cdot 10^{-1} \mathrm{~N} \mathrm{HCl}-8 \mathrm{~m}$ Harnstoff, 4. $1,99 \cdot 10^{-1} \mathrm{~N} \mathrm{HCl}-8 \mathrm{~m}$ Harnstoff, 5. 2,65 $10^{-1} \mathrm{~N} \mathrm{HCl}-$ 8 м Harnstoff.

Die Ausbeute an regenerierbarem $\mathrm{A}\left(\mathrm{SSO}_{3}{ }^{\ominus}\right)_{4}$ ist also von $56 \%$ beim Natriumversuch auf $67,5 \%$ beim Kontrollversuch in Ammoniak ohne Natrium gestiegen. Wir finden demnach, daß die Buntesalz-AKette bei Einwirkung von flüssigem Ammoniak nur geringfügig geschädigt wird, und daß eine 3,5 Min. lange Natriumbehandlung die Ausbeute an regenerierbarer $\mathrm{A}\left(\mathrm{SSO}_{3}{ }^{\ominus}\right)_{4}$-Fraktion nur geringfügig verringert.

\section{Elektrophoretisches Verhalten und Aminosäuren- Zusammensetzung der Fraktionen}

Die Fraktionen III - VI aus dem DEAE-Sephadex-Chromatogramm des Sulfitolyseproduktes von natriumbehandeltem $\mathrm{A}\left(\mathrm{SSO}_{3}{ }^{\ominus}\right)_{4}$ wurden elektro- phoretisch in Ameisensäure-Harnstoffpuffer ( $\mathrm{pH}$ 2,0 ) untersucht (Abb. 3).

Fraktion $V^{\prime}$ war die Hauptfraktion im DEAE-Sephadexchromatogramm des Sulfitolyseproduktes von nur mit Ammoniak behandeltem $\mathrm{A}\left(\mathrm{SSO}_{3}{ }^{\ominus}\right)_{4}$. Auch laut Elektropherogramm handelt es sich um ungeschädigtes $\mathrm{A}\left(\mathrm{SSO}_{3}{ }^{\ominus}\right)_{4}$.

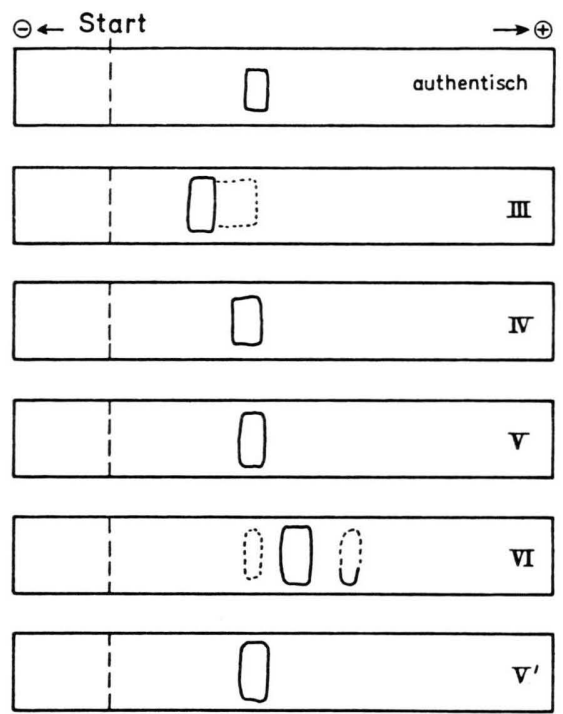

Abb. 3. Niederspannungs-Elektropherogramme (Ameisensäure-Harnstoff-Puffer, pH 2,0,8,2 V/cm). Die Banden III, IV, V und VI entsprechen den Fraktionen III, IV, V und VI in Abb. 1, Bande $\mathrm{V}^{\prime}$ entspricht Fraktion $\mathrm{V}^{\prime}$ in Abb. 2. Die Pherogramme wurden durch Besprühen mit $\mathrm{P}$ a u ly-Reagenz entwickelt.

Die DEAE-Sephadexfraktionen III, IV und V wurden nun der enzymatischen Hydrolyse mit Aminopeptidase-M sowie der salzsauren Hydrolyse unterworfen. Wie aus den Ergebnissen der Tab. 2,1 und 2,2 hervorgeht, stimmt die Aminosäuren-Zusammensetzung der Fraktion V bis auf den erhöhten Glutaminsäurewert mit der von Schweineinsulin$\mathrm{A}\left(\mathrm{SSO}_{3}{ }^{\ominus}\right)_{4}$ überein, während die Fraktionen III und IV Aminosäuren in abweichenden Molverhältnissen enthalten. Auffallend sind die niedrigen Werte für Leucin und Isoleucin für Fraktion III (Tab. 2,1).

Von besonderem Interesse sind die Abweichungen im $S$-Sulfocysteingehalt. Die langsamer wandernde Fraktion III enthält statt 4 nur 3 Cysteinreste. Schweineinsulin-A-Kette enthält kein Alanin. Fraktion III liefert mehr Alanin als Fraktion V. Erhöhte Alaninwerte in salzsauren Totalhydrolysaten von Natrium-behandelten A-Ketten wurden erstmalig von Katsoyannis ${ }^{9}$ festgestellt. Leider gibt es nur wenig 


\begin{tabular}{|c|c|c|c|c|c|}
\hline Aminosäure & $\begin{array}{l}\mathrm{DE} \\
\mathrm{Na} \\
\mathrm{III}\end{array}$ & $\begin{array}{l}\text {-Seph } \\
\text { Na } \\
\text { IV }\end{array}$ & $\begin{array}{c}\text { exfrak } \\
\text { Na } \\
\text { V }\end{array}$ & $\begin{array}{c}\text { nen } \\
\mathrm{NH}_{3} \\
\mathrm{~V}^{\prime}\end{array}$ & $\begin{array}{l}\text { Berech- } \\
\text { net für } \\
\text { Schweine- } \\
\text { insulin } \\
\mathrm{A}\left(\mathrm{SSO}_{3}\right)_{4}\end{array}$ \\
\hline \multicolumn{3}{|l|}{ S-Sulfo- } & 3,85 & 4,00 & \\
\hline $\begin{array}{l}\text { Asparagin- } \\
\text { säure }\end{array}$ & 0,15 & 0,11 & 0,13 & 0,07 & 0 \\
\hline $\left.\begin{array}{l}\text { Threonin } \\
\text { Serin } \\
\text { Asparagin } \\
\text { Glutamin }\end{array}\right\}$ & \multicolumn{4}{|c|}{$\begin{array}{l}\text { Bei } 48^{\circ} \text { in } 0,2 \text { M-Citrat-Puffer } \\
\text { wurden diese Aminosäuren } \\
\text { nicht vollständig getrennt. }\end{array}$} & $\begin{array}{l}1 \\
2 \\
2 \\
2\end{array}$ \\
\hline $\begin{array}{l}\text { Glutamin- } \\
\text { säure }\end{array}$ & 1,47 & 1,88 & 2,72 & 2,28 & 2 \\
\hline Glycin & 1,00 & 1,00 & 1,00 & 1,00 & 1 \\
\hline Alanin & 0,18 & 0,13 & 0,07 & 0,09 & 0 \\
\hline Cystin & 0,00 & 0,00 & 0,00 & 0,00 & 0 \\
\hline Valin & 0,84 & 1,08 & 0,97 & 1,34 & 1 \\
\hline Isoleucin & 0,97 & 1,50 & 1,52 & 1,93 & 2 \\
\hline Leucin & 1,03 & 1,53 & 1,80 & 2,05 & 2 \\
\hline Tyrosin & 1,61 & 1,29 & 1,97 & 2,04 & 2 \\
\hline
\end{tabular}

Tab. 2.1. Molverhältnis der Aminosäuren in AminopeptidaseM-Hydrolysaten. Na, Fraktionen aus dem mit Natrium in flüs. sigem Ammoniak behandelten $\mathrm{A}\left(\mathrm{SSO}_{3}{ }^{\ominus}\right)_{4} \cdot \mathrm{NH}_{3}$, Fraktion $\mathrm{V}^{\prime}$ des nur mit flüssigem Ammoniak behandelten $\mathrm{A}\left(\mathrm{SSO}_{3}{ }^{\ominus}\right)_{4}$.

\begin{tabular}{|c|c|c|c|c|}
\hline \multirow{3}{*}{ Aminosäure } & \multicolumn{3}{|c|}{$\begin{array}{l}\text { DEAE-Sephadex- } \\
\text { fraktionen }\end{array}$} & \multirow{3}{*}{$\begin{array}{l}\text { Berechnet } \\
\text { für } \\
\text { Schweine- } \\
\text { insulin } \\
\mathrm{A}\left(\mathrm{SSO}_{3}^{-}\right)_{4}\end{array}$} \\
\hline & $\mathrm{Na}$ & $\mathrm{Na}$ & $\mathrm{Na}$ & \\
\hline & III & IV & $\mathrm{V}$ & \\
\hline Cysteinsäure & 0,69 & 0,98 & 0,40 & 0 \\
\hline Asparaginsäure & 1,39 & 1,69 & 1,97 & 2 \\
\hline Threonin & 0,61 & 0,74 & 0,77 & 1 \\
\hline Serin & 1,25 & 1,57 & 1,51 & 2 \\
\hline Glutaminsäure & 3,23 & 3,54 & 3,98 & 4 \\
\hline Glycin & 1,00 & 1,00 & 1,00 & 1 \\
\hline Alanin & 0,25 & 0,23 & 0,16 & 0 \\
\hline Cystin & 0,71 & 0,89 & 1,58 & 2 \\
\hline Valin & 0,54 & 0,39 & 0,49 & 1 \\
\hline Isoleucin & 0.73 & 0,64 & 1,05 & 2 \\
\hline Leucin & 1,43 & 1,47 & 2,02 & 2 \\
\hline Tyrosin & 0,98 & 1,27 & 1,75 & 2 \\
\hline
\end{tabular}

Tab. 2.2. Molverhältnis der Aminosäuren in salzsauren Hydrolysaten, unkorrigiert.

Daten über die Abbauprodukte bei der Natriumbehandlung der einzelnen Peptidfragmente der AKette, so daß eine Erklärung der hier gefundenen veränderten Aminosäurezahlen nicht möglich ist (vgl. hierzu BENISEK et al. ${ }^{19}$ ).

\section{Die Insulin-bildende Potenz der Fraktionen}

Das entscheidende Kriterium für die Qualität der einzelnen an DEAE-Sephadex erhaltenen Fraktionen ist die Potenz zur Insulinbildung bei der Kombina- tion mit $\mathrm{B}\left(\mathrm{SSO}_{3}{ }^{\ominus}\right)_{2}$. Ein ungeschädigtes A-KettenPräparat sollte ebensoviel Insulin liefern wie eine schonend aus Insulin isolierte $S$-Sulfonat-A-Kette. Für diese Vergleichsversuche entschieden wir uns für das A-Kettenüberschußverfahren von Du et al. ${ }^{12}$, $\mathrm{da}$ es einfach ist und für einen Kombinationsversuch $10-20$ mg Kettengemisch ausreichen. In Tab. 3 sind die Insulingehalte der Oxydations-Produkte sowie die Insulin-Ausbeuten bezogen auf das Gemisch von A- und B-Kette, von dem ausgegangen wird, in den letzten beiden Spalten angegeben.

Aus Schweineinsulin isoliertes $\mathrm{A}\left(\mathrm{SSO}_{3}{ }^{\ominus}\right)_{4}$ liefert nach gemeinsamer Reduktion mit $\mathrm{B}\left(\mathrm{SSO}_{3}{ }^{\ominus}\right)_{2}$ und anschließender Autoxydation bei pH 10,6 ein Oxydationsprodukt mit 7,7\% Insulin (Mittel von 15 Versuchen, vgl. Versuchsteil). Die Insulinausbeute beträgt 6,5 Prozent. Diese Insulingehalte bzw. -ausbeuten werden nur von den an DEAE-Sephadex erhaltenen Fraktionen $\mathrm{V}$ und $\mathrm{V}^{\prime}$ erreicht. Das rohe Sulfitolyseprodukt des natriumbehandelten $\mathrm{A}\left(\mathrm{SSO}_{3}{ }^{\ominus}\right)_{4}$ ist im Kombinationsversuch 3 -4-mal weniger wirksam. Fraktion III besitzt sogar nur noch $5 \%$ der Insulin-bildenden Potenz des $\mathrm{A}\left(\mathrm{SSO}_{3}{ }^{\ominus}\right)_{4}$.

Schon die Behandlung von $\mathrm{A}\left(\mathrm{SSO}_{3}{ }^{\ominus}\right)_{4}$ mit flüssigem Ammoniak erniedrigt die Insulinausbeute beim nicht fraktionierten Sulfitolyseprodukt auf 3,0 Prozent.

\section{Zusammenfassende Diskussion}

Wir haben gezeigt, daß eine Buntesalz-Insulin-AKette durch Behandlung mit flüssigem Ammoniak nur wenig geschädigt wird, da man aus dem Reaktionsprodukt fast $70 \% \mathrm{~A}\left(\mathrm{SSO}_{3}{ }^{\ominus}\right)_{4}$ wieder isolieren kann.

Hat man die Buntesalz-A-Kette mit Natrium in flüssigem Ammoniak behandelt und anschließend der oxydativen Sufitolyse unterworfen, so lassen sich $56 \% \mathrm{~A}\left(\mathrm{SSO}_{3}{ }^{\ominus}\right)_{4}$ regenerieren. Der Vergleich dieser Ausbeuten mit den viel niedrigeren Werten von $10-20 \%$, die wir erhielten, als synthetische $S$-Benzyl-A-Kette mit Natrium in flüssigem Ammoniak behandelt wurde ${ }^{5}$, zeigt, daß das Vorliegen der schwer reduzierbaren Benzylthioäther-Gruppen und nicht die Aminosäurensequenz der A-Kette ein Hauptgrund für die niedrigen Ausbeuten an $\mathrm{A}\left(\mathrm{SSO}_{3}{ }^{\ominus}\right)_{4}$ ist.

19 W. F. Benisek, M. A. Raftery u. R.D. Cole, Biochemistry 6, 3780 [1967]. 


\begin{tabular}{|c|c|c|c|c|c|c|c|}
\hline \multirow{3}{*}{$\begin{array}{l}\text { Prä- } \\
\text { parat } \\
\text { Nr. }\end{array}$} & \multirow{3}{*}{$\begin{array}{l}\text { A-Ketten- } \\
\text { Präparat }\end{array}$} & \multirow{3}{*}{$\begin{array}{c}\text { Oxydations- } \\
\text { produkt } \\
{[\mathrm{mg}]}\end{array}$} & \multirow{2}{*}{\multicolumn{4}{|c|}{$\begin{array}{l}\text { Unkorrigierter Insulingehalt } \\
\text { im Oxydationsprodukt }\end{array}$}} & \multirow{3}{*}{$\begin{array}{c}\text { Insulin- } \\
\text { ausbeute } \\
{[\%]^{* *}}\end{array}$} \\
\hline & & & & & & & \\
\hline & & & {$[\%]^{* *}$} & $\begin{array}{l}\mathrm{E} / \mathrm{mg} \\
\text { Substanz }\end{array}$ & \%* & $\begin{array}{l}\text { Mittel- } \\
\text { wert }[\%]\end{array}$ & \\
\hline $\mathrm{S} 4$ & $\begin{array}{l}\mathrm{A}\left(\mathrm{SSO}_{3} \ominus\right)_{4} \\
\text { unbehandelt }\end{array}$ & \multicolumn{3}{|c|}{15 Oxydationsprodukte Tab. 1} & & 7,7 & 6,5 \\
\hline $\mathrm{S} 4$ & $\mathrm{~A}\left(\mathrm{SSO}_{3}{ }^{\ominus}\right)_{4} \mathrm{mit}$ & 10,9 & 77,8 & 0,55 & 2,20 & & \\
\hline S 18 & flüssigem $\mathrm{NH}_{3}$ & 10,6 & 75,7 & 1,43 & 5,72 & 3,8 & 3,0 \\
\hline $\mathrm{S} 20$ & behandelt & 11,8 & 84,9 & 0,87 & 3,48 & & \\
\hline S 31 & Fraktion $\mathrm{V}^{\prime}$ & 11,3 & 81,3 & 3,26 & 13,0 & & \\
\hline $\mathrm{S} 36$ & & 12,9 & 92,2 & 2,09 & 8,26 & 9,3 & 8,0 \\
\hline S 38 & & 12,6 & 90,0 & 1,61 & 6,44 & & \\
\hline S 7 & $\mathrm{~A}\left(\mathrm{SSO}_{3}{ }^{\ominus}\right)_{4} \mathrm{mit}$ & 11,4 & 82,0 & 0,1 & 0,4 & & \\
\hline S 9 & Natrium in flüssigem & 11,6 & 82,9 & 0,7 & 2,8 & 2,4 & 1,9 \\
\hline S 12 & $\begin{array}{l}\text { Ammoniak be- } \\
\text { handelt, roh }\end{array}$ & 11,4 & 82,0 & 0,98 & 3,92 & & \\
\hline S 24 & Fraktion III & $5,3 * * *$ & 58,8 & 0,067 & 0,26 & 0,5 & 0,3 \\
\hline S 6 & & 8,8 & 62,8 & 0,21 & 0,84 & & \\
\hline S 26 & Fraktion IV & $6,5 * * *$ & 71,7 & 0,58 & 2,32 & 2,3 & 1,6 \\
\hline S 14 & Fraktion V & 11,5 & 82,2 & 0,85 & 3,4 & & \\
\hline S 16 & & 9,9 & 71,2 & 1,90 & 7,2 & & \\
\hline S 22 & & 107 & 77,0 & 5,20 & 20,4 & 9,3 & 7,1 \\
\hline S 34 & & 10,8 & 77,7 & 1,62 & 6,48 & & \\
\hline
\end{tabular}

Tab. 3. Insulinausbeuten bei der Kombination von $\mathrm{B}\left(\mathrm{SSO}_{3}{ }^{\ominus}\right)_{2}$ aus Schweineinsulin mit $\mathrm{A}\left(\mathrm{SSO}_{3}{ }^{\ominus}\right)_{4}$ und Produkten, die nach Natriumbehandlung erhalten wurden. * Bezogen auf Schweineinsulin mit $25 \mathrm{E} / \mathrm{mg}$. ** Bezogen auf die Einwaage von 8,1 mg A-Kettenpräparat $+7,1 \mathrm{mg} \mathrm{B}\left(\mathrm{SSO}_{3} \odot\right)_{2}$ bzw. die abweichendenEinwaagen bei ***. *** Hier wurden $5,3 \mathrm{mg}$ A-Kettenpräparat mit $4,6 \mathrm{mg} \mathrm{B}\left(\mathrm{SSO}_{3}{ }^{\ominus}\right)_{2}$ kombiniert.

Das zweite Ergebnis dieser Arbeit ist der Befund, daß eine Inhibierung der Insulinbildung aus den Ketten erfolgt, falls die A-Kette nicht rein vorliegt, sondern teilentschwefelte A-Kettenprodukte selbst in geringen Prozentsätzen beigemengt sind.

Sauberes $\mathrm{A}\left(\mathrm{SSO}_{3}{ }^{\ominus}\right)_{4}$ liefert z. B. nach Kombination mit $\mathrm{B}\left(\mathrm{SSO}_{3}{ }^{\ominus}\right)_{2}$ Insulinausbeuten von $7-8$ Prozent. Ein Rohpräparat mit einem Anteil an $\mathrm{A}\left(\mathrm{SSO}_{4}{ }^{\ominus}\right)_{4}$ von immerhin beinahe $60 \%$ ergibt jedoch nur noch eine Insulinausbeute von 1,9 Prozent. Die Ausbeute ist also nicht auf etwa $2 / 3$, sondern auf $1 / 4$ abgesunken. Niedrige Insulinausbeuten brauchen demnach kein Beweis dafür zu sein, daß eine A-Kettensynthese mißlungen ist. Möglicherweise enthält das rohe Sulfitolyseprodukt zwar erhebliche Mengen an $\mathrm{A}\left(\mathrm{SSO}_{3}{ }^{\ominus}\right)_{4}$, die aber wegen des Inhibierungseffektes nur wenig Insulin bilden. Man muß also synthetische A-Kettenpräparate so wirksam reinigen, daß intaktes $\mathrm{A}\left(\mathrm{SSO}_{3}{ }^{\ominus}\right)_{4}$ in Substanz isoliert wird. Diese Präparate sollten bei der Kombination mit $\mathrm{B}\left(\mathrm{SSO}_{3}{ }^{\ominus}\right)_{2}$ dieselbe Insulinausbeute wie isolier- tes $\mathrm{A}\left(\mathrm{SSO}_{3}{ }^{\ominus}\right)_{4}$ liefern. Man wird dann die niedrigen Insulinausbeuten, die man beobachtet, wenn man synthetische Ketten mit natürlichen vergleicht, nicht mehr auf eine unvermeidliche Natriumschädigung zurückführen können. Vielmehr muß man fordern, daß die synthetischen Buntesalzketten der A- oder B-Kette isolierten Buntesalzketten in ihrer Insulinbildenden Potenz äquivalent sind.

Eine ähnliche Untersuchung über die Fraktionierung von rohen Sulfitolyseprodukten isolierter und synthetischer B-Kette mit dem Ziele festzustellen, ob und in welchen Ausbeuten voll wirksames $\mathrm{B}\left(\mathrm{SSO}_{3}{ }^{\ominus}\right)_{2}$ auch dann zu isolieren ist, wenn die Kette eine $\mathrm{Na}$ triumbehandlung mitgemacht hat, steht noch aus.

Wir danken dem Nordisk Insulinlaboratorium, Gentofte, Dänemark, für die Überlassung kristallinen Schweineinsulins, der Arbeitsgemeinschaft industrieller Forschungsvereinigungen für die Unterstützung des Forschungsvorhabens 1318, und dem Landesamt für Forschung beim Ministerpräsidenten des Landes Nordrhein-Westfalen. 